\title{
All-optical storage and processing in optical fibers
}

\author{
Luc Thévenaz, Nikolay Primerov, Sanghoon Chin, Yair Antman*, Andrey Denisov, \\ Avi Zadok*, Marco Santagiustina** \\ EPFL Swiss Federal Institute of Technology, Institute of Electrical Engineering, SCI-STI-LT, \\ Station 11, 1015 Lausanne, Switzerland \\ *Faculty of Engineering, Bar-Ilan University, Ramat-Gan 52900 Israel \\ **CNIT-Department of Information Engineering, University of Padova, via Gradenigo 6b, 35131, Padova, Italy \\ Author e-mail address: Luc.Thevenaz@EPFL.CH \\ (Keynote Address)
}

\begin{abstract}
The recent possibility to generate and read dynamic Bragg gratings in optical fibers by the interaction of multiple optical waves through stimulated Brillouin scattering has opened a new field to realize all-optical fiber-based functions.
\end{abstract}

OCIS codes: (060.4370) Nonlinear optics, fibers; (290.5900) Scattering, stimulated Brillouin; (070.4340) Nonlinear optical signal processing

The generation of dynamic Bragg gratings by the interaction of 2 optical waves through stimulated Brillouin scattering has offered the possibility to implement alloptical functions that were so far impossible to realize in optical fibers. Using this feature a local grating can be placed at any position along an optical fiber and can be repositioned dynamically with a total flexibility through a purely optical interaction. This new tool has found direct applications in all-optical delay lines, demonstrating a tunable delaying capacity of more than 1 microsecond with an ideal reconfiguration time. But it turns out that its field of application is much vaster, from optical storage and analog operations on the signal (time reversal, derivative, integration, dynamic filtering, ...) to distributed sensing with an extreme spatial resolution.

\section{PRINCIPLE}

The generation of dynamic Brillouin gratings is based on the following principle [1]: two intense optical waves counterpropagating - hereafter called "pumps" - along the slow axis of a polarization maintaining fiber can create a localized acoustic grating (Brillouin dynamic grating) at a given point using SBS if they are properly modulated (e.g. optical pulses), as shown in Fig. 1. Since the created Brillouin dynamic grating is longitudinal, it can reflect a signal pulse with polarization along the orthogonal axis if the frequency of the signal satisfies the Bragg condition imposed by the grating. In a PM fiber refractive indices are different for the two orthogonal states of polarization, leading for a given physical pitch of the Brillouin dynamic grating to two different optical pitches for slow and fast axes. This leads to a shift of the grating resonance for the fast axis to another optical frequency. This way it is possible to reflect a signal pulse polarized along the fast axis with the grating created by optical waves along the slow axis and to entirely decorrelate the processes of grating dynamic generation and signal reflection. The frequency difference between the resonances for the two orthogonal axes is fixed by the local birefringence of the fiber.

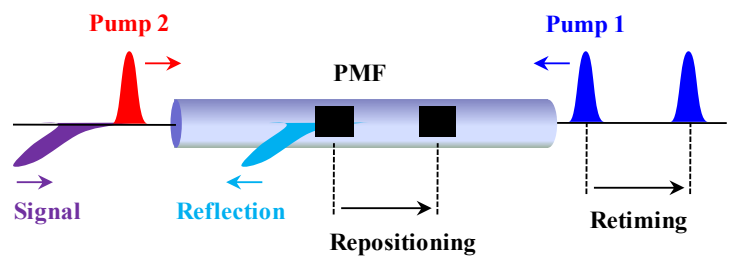

Fig. 1. Principle to generate a dynamic Brillouin grating (DBG) in a polarization maintaining fiber, based on the ordinary process of stimulated Brillouin scattering, and to realize a localized movable reflection of an orthogonally polarized signal wave.

If the two intense optical waves are both formed of isolated pulses, the acoustic wave is generated during the overlapping time and then rapidly decays with a time constant $\tau_{a}=11 \mathrm{~ns}$ given by the acoustic loss. The dynamic grating is therefore temporary and can only reflect for several nanoseconds. This kind of dynamic grating generation is therefore only appropriate for reflecting signals of short durations. Several techniques have been proposed to make the grating permanent, or at least extend its duration: the simplest is to use pulse trains for the grating generating waves, so that the grating is refreshed before it significantly decays $[2,3]$. This technique shows an intrinsic limit for the maximum fiber length over which the grating can be freely moved, that is given by the pulses repetition rate. If this condition is not fulfilled, a second grating - or more gratings - may be generated in the fiber. Another approach is based on a frequency correlation technique, in which the two intense optical waves are identically frequency dithered [4]. As a result of their counterdirectional propagation, they show a constant frequency difference at specific evenly spaced locations. At these specific locations the acoustic wave can steadily grow, while the frequency dithering washes out the grating in between. The problem related to this approach is that the central frequency of the grating is also dithering, requiring the reflected signal to be identically frequency- dithered that is inconvenient in most real situations. The product between the free moving temporal range of the grating and its bandwidth is also intrinsically limited to $\sim 300$. It means that a $10 \mathrm{GHz}$ signal cannot be delayed over more than $30 \mathrm{~ns}$. Very recently another correlation technique was proposed, based on identical random phase modulations over the 2 intense optical waves generating the grating [5, 6]. Again the phase difference between the 2 waves will be steadily constant at specific evenly spaced locations, with a separation given by the periodicity of the random sequence. Using a pseudo-random bit sequence generator, this 
periodicity can be made arbitrarily long and the product of the free temporal range by the bandwidth can exceed $10^{9}$. This is a clear advantage of this approach that also benefits from the fact that the central frequency of the grating is steadily constant. This technique suffers anyway from the penalty of a significant noise due to transient partially building gratings all over the fiber.

It must be mentioned that recent works have realized and interrogated dynamic Brillouin gratings in standard single mode fibers, i.e. non-polarization maintaining fibers, using orthogonally polarized lightwaves for the generation and the interrogation [6-8].

The reflection properties of the dynamic gratings can be properly described by identifying such gratings to very weak Bragg gratings: the peak reflectivity scales as the grating length squared, while the bandwidth is inversely proportional to the same length [9]. So the price to be paid for a larger bandwidth is a much reduced reflectivity. When the grating is generated by pulses or a pseudo random bit sequence, the bandwidth is essentially given by the pulse width or the symbol duration, as illustrated in Fig. 2. Apart from the fact that the gratings can be dynamically positioned at any preset position along the fiber, their length can be also arbitrarily chosen and, unlike classical static fiber Bragg gratings, and can be made extremely long, over many tens or hundreds of meters. A fully distributed reflection can be obtained, as shown in Fig. 2, and an extremely narrow grating bandwidth can be achieved [7, 9].

Hereafter some typical applications of dynamic Brillouin gratings are presented, to illustrate the large - but still widely unexplored - potentialities of this new tool.
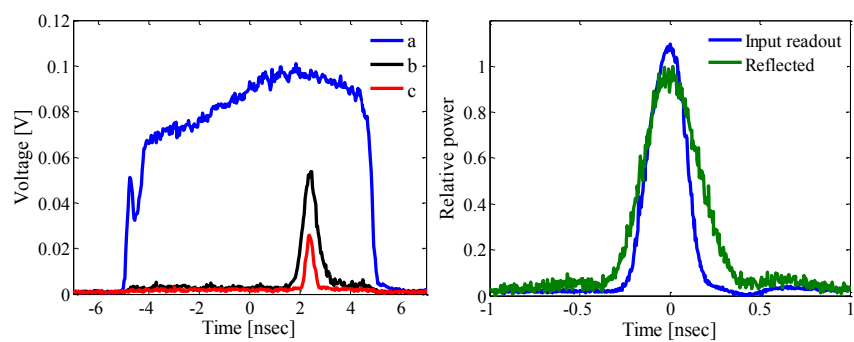

Fig. 2: Left: Reflected waveforms from the dynamic Brillouin grating, interrogated by 260 ps long, isolated periodic pulses. a) - dynamic grating written by CW pumps. b) both pumps modulated by a pseudo-random bit sequence with symbol duration $\mathrm{T}=1 \mathrm{~ns}$. c) Same as b) with $\mathrm{T}=167 \mathrm{ps}$.

Right: Input (blue) and readout pulses (green) reflected by the dynamic Brillouin grating generated by a pseudo-random bit sequence with symbol duration $\mathrm{T}=167 \mathrm{ps}$.

\section{ALL OPTICAL TUNEABLE DELAY LINE}

Since reflecting gratings are created at any position along the fiber, a signal pulse can be also reflected at different points, thus showing a delay due to the time-offlight for the back-reflected signals $[3,10,11]$.

Since the acoustic grating can only be built up where the SBS interaction between the two pump pulses takes place, it can be flexibly created at any preset position over the entire length of the HiBi fiber, simply by retiming Pump 1 pulse. Using this principle an isolated signal pulses with FWHM duration of 650 ps was continuously delayed up to $1.15 \mu \mathrm{s}$ through a $120 \mathrm{~m}$ long PMF, equivalent to a fractional delay of 1769 , as shown in Fig. 3. This system is also suitable for a real data bit sequence, using the techniques described above for the generation of permanent localized dynamic gratings with their inherent limitations.

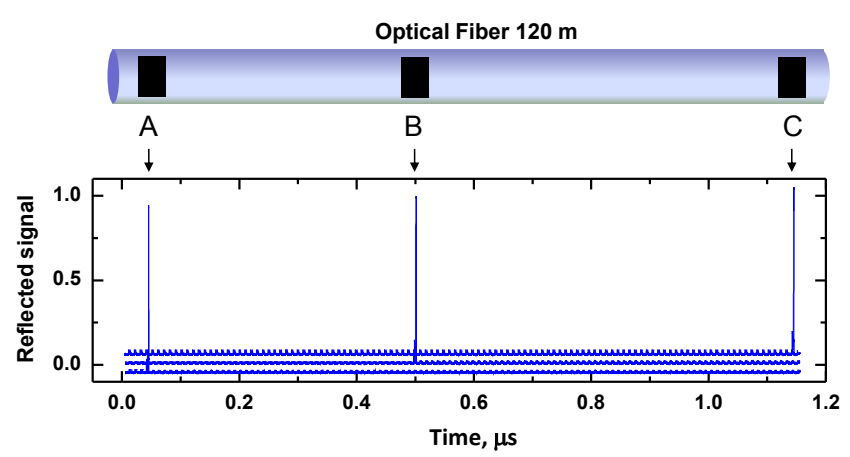

Fig. 3: Reflected pulses on dynamic Brillouin gratings positioned at 3 successive positions (A,B,C) along a $120 \mathrm{~m} \mathrm{HiBi}$ fiber. 650 ps pulses could be delayed by more than $1 \mu$ s with minor distortion.

\section{STORAGE AND TIME REVERSAL OF AN OPTICAL SIGNAL}

The acoustic grating generated by the modulated pumps turns out to have a spatial distribution given by the convolution between the 2 pumps. Different operations can be realized using this property and the result can be read using the reflected signal. For instance an optical signal can be stored and retrieved at a later time when one of the pumps is the signal to be stored and a very short pulse is used for the other pump. This way the generated acoustic wave is an exact spatial replica of the signal to be stored and the material vibration remains static in the fiber. It can be read at a later time using a very short orthogonally polarized reading pulse reflected on this acoustic wave. If this reading pulse is launched from the opposite end of the fiber, a timereversed copy of the input signal is retrieved [12], as shown in Fig. 4.
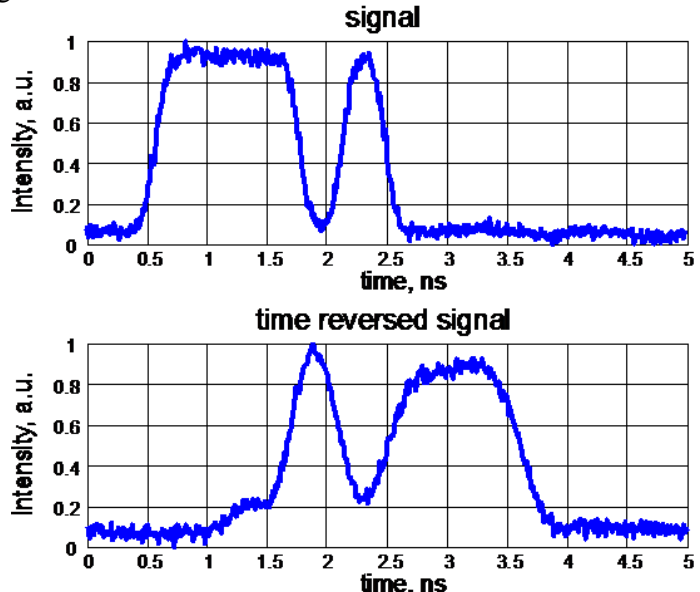

Fig. 4. Time reversed copy of a data sequence obtained by storing the signal in a static acoustic vibration in the fiber and retrieved using a signal launched in the opposite direction. The decaying amplitude of the retrieved signal is due to the finite acoustic damping time in the silica (typ. $10 \mathrm{~ns}$ ).

\section{OPTICAL CALCULUS: INTEGRATION AND DIFFERENTIATION}

By convolving the input signal modulating one of the pumps with a variety of different temporal waveform for the other pump, other operations can be realized, such as optical integration and differentiation [13]. Optical signal 
integration with dynamic Brillouin gratings is simply sketched in the diagram shown in Fig. 5. First, a dynamic Brillouin grating is uniformly created in a sufficiently long fiber, on which the orthogonally polarized probe signal to be integrated is backreflected. The reflected waveform represents the time integral of the complex envelope of the input probe waveform. The principle was experimentally tested using various optical waveforms. Fig. 6 (left) depicts the optical integration of a single $800 \mathrm{ps}$ pulses; the agreement with numerical values is excellent. The integration time window in the experiment amounts to $T=2 n L / c \approx 4$ ns.

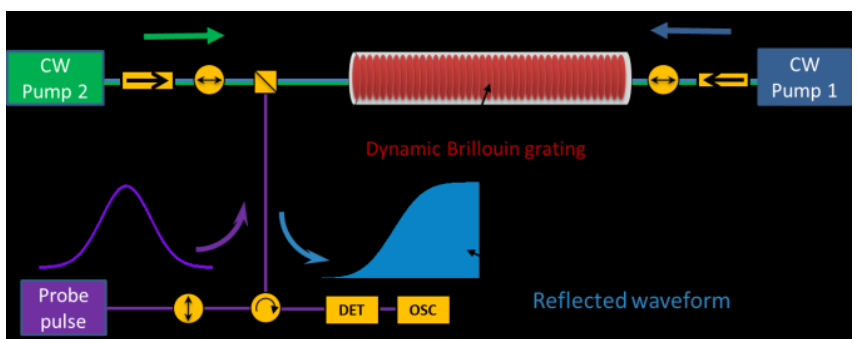

Fig. 5. Setup for optical signal integration based on dynamic Brillouin gratings. A long weak distributed reflector is so created in the fiber that reflects the fraction of the signal present in the grating section. PBC polarization beam combiner; DET - detector; OSC - oscilloscope.
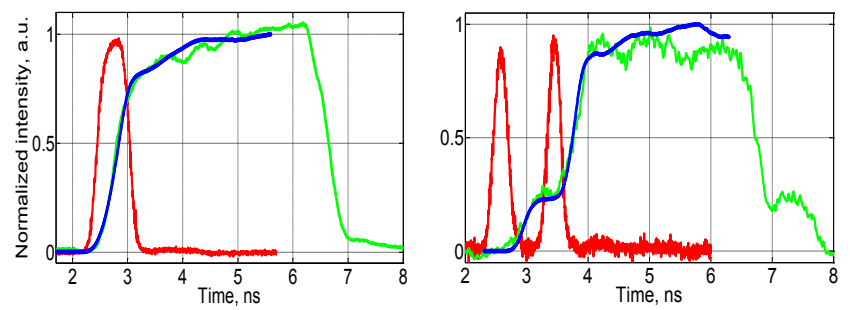

Fig. 6. Experimentally measured input and output waveforms experiencing optical signal integration. Left: $800 \mathrm{ps}$ pulse; Right: two 300ps in-phase pulses delayed by $900 \mathrm{ps}$. Input waveforms (red), experimentally obtained integration (green), numerically calculated integration (blue).

Then Fig. 6 (right) represents the integration of a nearly symmetric double pulse input consisting of two in-phase pulses (FWHM 300 ps, delayed by 900 ps). The agreement with numerical integration is good. It must be pointed out that the integral over the field amplitude of the double pulse is twice the integral over a single pulse, while the measured intensity is equal to the squared field integral. Consequently the second step in the integrated waveform must be three times higher than the first step, fully confirmed by the experimental trace shown in Fig. 6 (right).

This way it is clear that the proposed integrator operates coherently on the complex amplitude of the input optical waveform.

Optical signal differentiation is a particular case of optical storage, in which the difference between 2 slightly delayed replicas of the input waveform is performed using a specially shaped reading waveform. This can be realized in two stages, just like the optical storage, as illustrated by the experimental setup being shown in Fig. 7 (top): firstly the optical data waveform to be differentiated is first stored in the dynamic Brillouin grating; then a reading waveform, consisting of two short pulses with a relative $\pi$ phase difference, is launched along the orthogonal polarization into the fiber. The process can be also viewed as the interference of two reflected $\pi$-phase shifted replicas of the stored waveform, which are thus of opposite amplitude and slightly delayed. This corresponds to the first order time derivative of the input waveform. Fig. 7 (bottom) shows the all-optical signal differentiation of a $7 \mathrm{~ns}$ bell-shaped pulse. Here again the operation is performed coherently, on the signal amplitude, but only the intensity is detected that appears as a rectification of the signal.
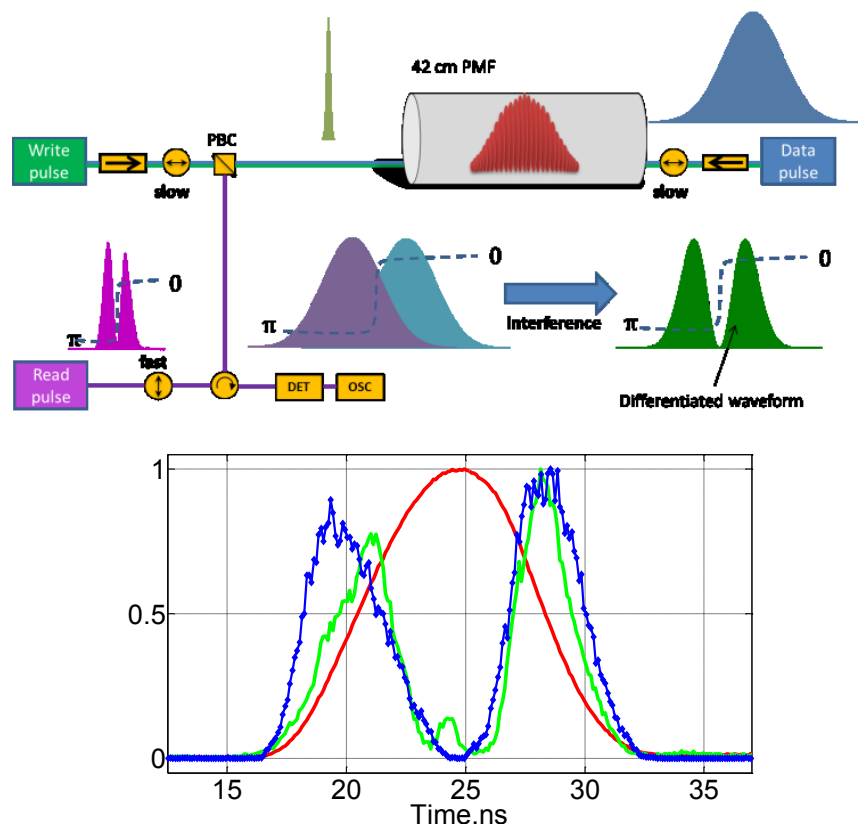

Fig. 7. Top: Schematic diagram of the setup performing optical signal differentiation.

Bottom: Experimentally measured input and output waveforms experiencing optical signal differentiation. Input waveform (red): $7 \mathrm{~ns}$ bellshaped input pulse, experimentally obtained differentiation (green), numerically calculated differentiation (blue). The results are measured in intensity and so equal to the rectified squared amplitude

\section{DISTRIBUTED SENSING}

When the 2 intense counterpropagating waves generating the grating are continuous, the acoustic wave extends all over the fiber length and its local amplitude is proportional to the local Brillouin gain in the fiber. By reflecting a very short pulse in the orthogonal polarization, a map of the local amplitude of the acoustic wave can be obtained in the time domain. By incrementally varying the frequency difference between the 2 intense counterpropagating waves and recording for each step the local amplitude of the acoustic wave, the local spectral distribution of the Brillouin can be retrieved and the local value of the Brillouin shift can be calculated [14]. It is wellknown that the Brillouin shift is very dependent on the temperature and the strain experienced by the fiber. A spatial resolution of $6 \mathrm{~mm}$ could be demonstrated using this system [15], as illustrated in Fig. 8. This is fully comparable to point sensors such as fibre Bragg gratings, however showing the great flexibility of a fully continuous 
distributed sensing, equivalent to many thousands of distinct point sensors and requiring no special fibre preparation. This will certainly cause a significant change in the appreciation of this type of sensors, by broadening the field of applications to small and medium-size structures.

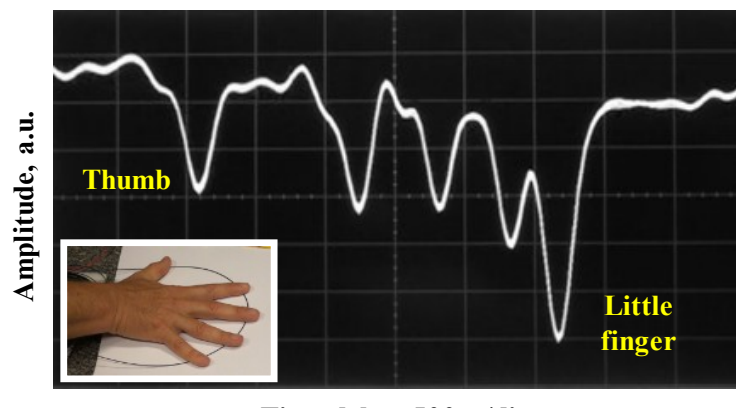

Time delay, 500ps/div

Fig. 8. Temporal distribution of the signal pulse reflection along the sensing fiber when 5 fingers from one hand are placed on the fiber, as shown in the inset image. The pump frequency difference is adjusted to give a maximal reflection at ambient temperature. The local heating of the fingers is sufficient to create enough detuning which results in a localized drop of the Brillouin grating reflectivity exactly at the fingers position.

\section{CONCLUSION AND PERSPECTIVES}

Through the mediation of an acoustic vibration in the material all-optical signal processing can be realized entirely in optical fibers. A non-exhaustive set of processing functions and applications have been presented, which are just the emerging part of a still uncovered vast field of possible realizations. For instance the generation of multiple gratings makes possible the realization of very flexible reconfigurable filters for microwave photonics [16]. It must be pointed out that all the functional processors presented here are realized using the same basic experimental configuration. This means that the system can be reconfigured to realize different operations by simply changing the temporal shaping of the interacting signals, without modifying the experimental layout. This tool will be probably an important building block for future all-optical signal processing functions.

\section{REFERENCES}

[1] K. Y. Song, W. Zou, Z. He, and K. Hotate, "Alloptical dynamic grating generation based on Brillouin scattering in polarization-maintaining fiber," Opt. Lett., vol. 33, pp. 926-928, 2008.

[2] S. Chin, N. Primerov, and L. Thevenaz, "Photonic delay line for broadband optical signals, based on dynamic grating reflectors in fibers," in 36th European Conference and Exhibition on Optical Communication (ECOC), Torino, 2010, p. 5621406.

[3] S. Chin and L. Thévenaz, "Tunable photonic delay lines in optical fibers," Laser \& Photonics Reviews, pp. n/a-n/a, 9 FEB 20122012.

[4] W. Zou, Z. He, K.-Y. Song, and K. Hotate, "Correlation-based distributed measurement of a dynamic grating spectrum generated in stimulated
Brillouin scattering in a polarization-maintaining optical fiber," Opt. Lett., vol. 34, pp. 1126-1128, 2009.

[5] Y. Antman, N. Primerov, J. Sancho, L. Thevenaz, and A. Zadok, "Localized and stationary dynamic gratings via stimulated Brillouin scattering with phase modulated pumps," Opt. Express, vol. 20, pp. 7807-7821, March 21, 20122012.

[6] A. Zadok, Y. Antman, N. Primerov, A. Denisov, J. Sancho, and L. Thevenaz, "Random-access distributed fiber sensing," Laser \& Photonics Reviews, vol. 6, pp. L1-L5, 2012.

[7] K. Y. Song, "Operation of Brillouin dynamic grating in single-mode optical fibers," Opt. Lett., vol. 36, pp. 4686-4688, 2011.

[8] K. Y. Song, "Effects of induced birefringence on Brillouin dynamic gratings in single-mode optical fibers," Opt. Lett., vol. 37, pp. 2229-2231, 2012.

[9] K. Y. Song and H. J. Yoon, "Observation of narrowband intrinsic spectra of Brillouin dynamic gratings," Opt. Lett., vol. 35, pp. 2958-2960, 2010.

[10] N. Primerov, S. Chin, K. Y. Song, and L. Thevenaz, "Ultra Wide Range Tunable Delay Line Using Dynamic Grating Reflectors in Optical Fibers," in Optical Fiber Communication Conference, San Diego, CA, 2010, p. OWF6.

[11] Y. Antman, N. Primerov, J. Sancho, L. Thevenaz, and A. Zadok, "Long Variable Delay and Distributed Sensing Using Stationary and Localized Brillouin Dynamic Gratings," in Optical Fiber Communication Conference (OFC), Los Angeles, California, 2012, p. JW2A.24.

[12] S. Chin, N. Primerov, K. Y. Song, L. Thevenaz, M. Santagiustina, and L. Ursini, "True Time Reversal via Dynamic Brillouin Gratings in Polarization Maintaining Fibers," in Nonlinear Photonics (NP), Karlsruhe, Germany, 2010, p. NThA6.

[13] N. Primerov, S. Chin, L. Thévenaz, L. Ursini, and M. Santagiustina, "All-optical calculus based on dynamic Brillouin grating reflectors in optical fibers," in Slow and Fast Light (SL), Toronto, Canada, 2011, p. SLMA3.

[14] K. Y. Song, S. Chin, N. Primerov, and L. Thevenaz, "Time-Domain Distributed Fiber Sensor With $1 \mathrm{~cm}$ Spatial Resolution Based on Brillouin Dynamic Grating," Journal of Lightwave Technology, vol. 28, pp. 2062-2067, 2010.

[15] S. Chin, N. Primerov, and L. Thevenaz, "SubCentimeter Spatial Resolution in Distributed Fiber Sensing Based on Dynamic Brillouin Grating in Optical Fibers," IEEE Sensors Journal, vol. 12, pp. 189-194, 2012.

[16] J. Sancho, N. Primerov, S. Chin, Y. Antman, A. Zadok, S. Sales, and L. Thévenaz, "Tunable and reconfigurable multi-tap microwave photonic filter based on dynamic Brillouin gratings in fibers," Opt. Express, vol. 20, pp. 6157-6162, February 29, 20122012. 
